\title{
FINITELY GENERATED NON-HOPFIAN GROUPS
}

\author{
A. H. RHEMTULLA ${ }^{1}$
}

\begin{abstract}
We discuss finitely generated groups that are badly non-Hopfian. Given any countable group $L$, we construct a finitely generated group $G=K \times H$ where $H$ is isomorphic to $G$ and $L$ is a two-step subnormal subgroup of $K$.
\end{abstract}

1.

1.1. Following P. Neumann, we call a group $G$ weakly Hopfian if $G=K \rtimes H$ and $H \simeq G$ imply $K=\{1\}$. In [3] he investigated weak Hopficity in finitely generated solvable groups and proved that every finitely generated nilpotent-bynilpotent group is weakly Hopfian but there exists a two generator solvable group of length three that is not weakly Hopfian. These results have motivated the investigations here. We call a group $G$ subnormally Hopfian if $G=K \rtimes H, H$ a subnormal subgroup of $G$ and $H \simeq G$ imply $K=\{1\} . G$ is directly Hopfian if $G=K \times H$ and $H \simeq G$ imply $K=\{1\}$. We write $G=K \rtimes H$ to mean that $G=K H, K \triangleleft G$ and $K \cap H=\{1\}$.

1.2. It turns out that if $G$ is a finitely generated group that is not subnormally Hopfian then $G / B$ is not directly Hopfian, where $B$ is the group generated by all abelian subnormal subgroups of $G$-the Baer radical of $G$. We shall discuss only finitely generated groups that are not subnormally Hopfian. Theorem 1 gives some properties of such a group $G$. These properties imply, in particular, that $G$ cannot be solvable. Theorem 2 deals with the result mentioned at the beginning of this paragraph. Theorems 3 and 4 deal with the embedding problems. Simple constructions and use of the beautiful results of $P$. Hall in [1] show that any countable group $L$ can be subnormally embedded in a finitely generated group that is not directly Hopfian. We have not tried to pursue this line to obtain results similar to those of J. M. Tyrer-Jones in [4].

2.

2.1. For a given group $G$ and positive integer $m, \gamma_{m}(G)$ will denote the $m$ th term of the lower central series of $G$. Thus $\gamma_{1}(G)=G$ and $\gamma_{m+1}(G)=\left[\gamma_{m}(G), G\right]$. Occasionally we shall write $G^{\prime}$ to denote $\gamma_{2}(G)$. We shall also use the symbol $\mathfrak{B}$ for the class of groups generated by abelian subnormal sufbgroups, $\mathfrak{A}$ for abelian groups, $\mathfrak{N}$ for nilpotent groups, $\mathbb{S}$ for finitely generated groups, $\max -\boldsymbol{n}$ for groups satisfying the maximal condition for normal subgroups and $\mathfrak{X} \mathfrak{R}$ for abelian-bynilpotent groups.

\subsection{Statements and proofs of the theorems.}

Received by the editors February 8, 1980.

1980 Mathematics Subject Classification. Primary 20E15, 20E22; Secondary 20 E34.

${ }^{1}$ Research partially supported by a grant from NSERC.

() 1981 American Mathematical Society $0002-9939 / 81 / 0000-0108 / \$ 01.75$ 
THEOREM 1. If $G=K \rtimes H \in \mathbb{S}, H \simeq G$ and $H$ subnormal in $G$ then the following hold.

(i) $\gamma_{m}(H)$ is normal in $G$ for some positive integer $m$.

(ii) $K=K^{\prime}$.

(iii) $K \cap M \in \mathfrak{B}$ where $M=H^{G}=\left\langle H^{g} ; g \in G\right\rangle$.

Proof. Since $H$ is subnormal in $G$ the $n$-fold commutator group $[K, H, \ldots, H]$ $<K \cap H=\{1\}$. Thus $\left[\gamma_{m}(H), K\right]=\{1\}$ for some positive integer $m$ [2, Theorem 3.8]. This gives (i).

Since \&S $\cap \mathfrak{X} \mathfrak{R} \subset \max -n \subset$ class of Hopfian groups, $K<\gamma_{2}\left(\gamma_{m}(G)\right)$. Therefore $\gamma_{m}(G)=K \times \gamma_{m}(H)$ and $\gamma_{2}\left(\gamma_{m}(G)\right)=K^{\prime} \times \gamma_{2}\left(\gamma_{m}(H)\right)>K$. Thus $K=K^{\prime}$. Let * denote the natural map of $G$ onto $G / \gamma_{m}(H)=G^{*}$. Then $K \simeq K^{*}$ and $H^{*}$ is nilpotent of class $m$ and subnormal in $G^{*}$. Let $B^{*}$ be the Baer radical of $G^{*}$. Then $H^{*}<B^{*} \triangleleft G^{*}$. Thus $M^{*}<B^{*}$ where $M=\left\langle H^{g} ; g \in G\right\rangle$. Thus $M^{*} \cap K^{*}<B^{*}$. But $M^{*} \cap K^{*} \simeq M \cap K$. Hence $M \cap K \in \mathfrak{B}$.

TheOREM 2. Suppose $G=K \rtimes H \in \mathbb{S}, H$ a subnormal subgroup of $G$ and $H \simeq G$. Let ${ }^{*}$ be the natural map of $G$ onto $G / B=G^{*}$ where $B$ is the Baer radical of $G$. Then $G^{*}=K^{*} \times H^{*}, H^{*} \simeq G^{*}$ and $K^{*} \neq\{1\}$ if $K \neq\{1\}$.

Proof. Let $M=\left\langle H^{g} ; g \in G\right\rangle$. By Theorem $1, K=K^{\prime}$ and $K \cap M \subseteq B$. Thus $K^{*}$ is a finitely generated perfect group. Since a nontrivial group cannot be perfect and nilpotent, $K \nsubseteq B$ unless $K=\{1\}$. That $H^{*} \triangleleft G^{*}$ follows from Theorem 1. Let $\theta$ be the isomorphism of $G$ onto $H$ then $\theta(B)$ is the Baer radical of $H$ and hence $\theta(B)=B \cap H$ and $G / B \simeq H / B \cap H \simeq H^{*}$. This completes the proof.

Suppose that $G=K \times H \in \mathbb{S}, H \simeq G$ and $K \neq\{1\}$. Write $K_{1}=K, H_{1}=H$. Since $H_{1} \simeq G, H_{1}=K_{2} \times H_{2}$ where $K_{2} \simeq K_{1}, H_{2} \simeq G$. More generally,

$$
G=K_{1} \times K_{2} \times \cdots \times K_{m} \times H_{m}, \quad H_{m} \simeq G, K_{i} \simeq K
$$

for all $i=1, \ldots, m$ and $m=1,2, \ldots$ Since $G=\left\langle g_{1}, \ldots, g_{n}\right\rangle$ for a suitable choice of generators $g_{i} \in G, g_{i}=k_{i 1} k_{i 2} \cdots k_{i m} h_{i m}$ where $k_{i j} \in K_{j}, h_{i m} \in H_{m}$. Moreover this representation is unique. Thus for all $i=1, \ldots, n, j=1,2, \ldots$, $k_{i j}=w_{i j}\left(g_{1}, \ldots, g_{n}\right)=w_{i j}\left(k_{1 j}, \ldots, k_{n j}\right)$ and $w_{i j}\left(k_{1 l}, \ldots, k_{n l}\right)=1$ if $l \neq j$. Let $\phi_{j}$ be the isomorphism of $K_{j}$ onto $K$. Then $K=\left\langle k_{1 j} \phi_{j}, \ldots, k_{n j} \phi_{j}\right\rangle, j=1,2, \ldots$, and so

$$
w_{i l}\left(k_{1 j} \phi_{j}, \ldots, k_{n j} \phi_{j}\right)= \begin{cases}1, & \text { if } j \neq l, \\ k_{i j}, & \text { if } j=l .\end{cases}
$$

This means that $K$ has infinitely many generating sets $S_{j}=\left\{k_{1 j} \phi_{j}, \ldots, k_{n j} \phi_{j}\right\}$ and words $w_{i l}$ satisfying (*). These are nonisomorphic sets in the sense that no automorphism of $K$ can map $S_{j}$ onto $S_{l}$ as ordered sets. A simple way to construct such a group is to take $K$ to be a finitely generated simple group containing a wreath product $X$ wr $T$ where $X$ is any nonabelian group and $T=\langle t\rangle$ is infinite cyclic. Let $a, b \in X$ such that $[a, b]=c \neq 1$. Write $x_{i}$ to denote $t^{-i} x t^{i}, x \in X$. We can take $K$ to be a two-generator group as shown by Wilson in [5, p. 20], and we can take $X$ to be a nonabelian finite $p$-group so that $X$ wr $T$ is linear. Suppose that $K=\langle x, y\rangle$. Let $C$ be the cartesian product of copies $K_{i}$ of $K, i \in \mathbf{N}$; let $G$ be the 
subgroup of $C$ generated by $\underline{x}=(x, x, \ldots), y=(y, y, \ldots)$ and $\tau=$ $\left(1, t, t^{2}, \ldots\right)$. Then the diagonal subgroup $\Delta$ of $C$ is contained in $G$ and if we denote by $g$ the element $(g, g, \ldots)$ of $\Delta$, then $\left[\underline{a}^{\tau}, \underline{b}\right]=([a, b], 1,1, \ldots)$. Since $K$ is simple, $\bar{K}_{1} \leqslant G$. Similarly $K_{i}$ and hence the direct product of $K_{i}, i \in \mathbf{N}$, is a subgroup of $G$. Let $H_{1}=\left\langle\underline{x}^{\prime}, \underline{y}^{\prime}, \tau^{\prime}\right\rangle$ where $\underline{x}^{\prime}=(1, x, x, \ldots), \underline{y}^{\prime}=(1, y, y, \ldots)$ and $\tau^{\prime}=\tau \underline{t}^{\prime-1}=\left(1,1, t, t^{2}, \ldots \overline{)}\right.$. Then $H_{1}<G, H_{1} \simeq G$, and $G \overline{=} K_{1} \times H_{1}$. Thus we have shown

THEOREM 3. There exists a three-generator group $G$ that is not directly Hopfian.

Now let $K=\langle x, y\rangle$ be as above and let $K^{*}=\left\langle x^{*}, y^{*}\right\rangle$ be a copy of $K$. If $L$ is any countable group, then by Theorem $A$ of [1], there exists a group $J=\left\langle K, K^{*}\right\rangle$ such that $L$ is a two-step subnormal subgroup of $J$. Let $C$ be the cartesian product of copies $J_{i}, i \in \mathbf{N}$, of $J$ and let $\Delta$ denote the diagonal subgroup of $C$. Then $\Delta=\left\langle\underline{x}, y, \underline{x}^{*}, \underline{y}^{*}\right\rangle$ where $g=(g, g, \ldots)$ for all $g \in J$. The subgroup $G=$ $\left\langle\Delta, \tau, \tau^{*}\right\rangle$ where $\tau=\left(1, t, t^{2}, \ldots\right)$ and $\tau^{*}=\left(1, t^{*}, t^{* 2}, \ldots\right)$ is the direct product of $J_{1}$ and $H_{1}$ where $H_{1}=\left\langle\Delta^{\prime}, \tau^{\prime}, \tau^{*^{\prime}}\right\rangle$. Here $\Delta^{\prime}$ is generated by $g^{\prime}=(1, g, g, \ldots)$, $g \in J$, and $\tau^{\prime}=\tau \underline{t}^{\prime-1}=\left(1,1, t, t^{2}, \ldots\right) . \tau^{* \prime}$ is defined similarly. It is easy to see that $J_{1}<G$ for $\left[\underline{a}^{\tau}, \underline{b}\right]=([\underline{a}, \underline{b}], 1,1, \ldots)$ and since $K$ is simple, $\left\langle[\underline{a}, \underline{b}]^{\Delta}\right\rangle<$ $\{(k, 1,1, \ldots) ; k \in K\}$. Similarly using $\underline{a}^{*}, \underline{b}^{*}$ and $\tau^{*}$ we get $G>\left\{\left(k^{*}, 1,1, \ldots\right)\right.$; $\left.k^{*} \in K^{*}\right\}$. Since $J=\left\langle K, K^{*}\right\rangle, G \geqslant J_{1}$. The rest follows easily from this and we have the following final result.

THEOREM 4. Let $L$ be any countable group. Then there exists a six-generator group $G=K \times H$ such that $H \simeq G$ and $L$ is a two-step subnormal subgroup of $K$.

\section{REFERENCES}

1. P. Hall, On the embedding of a given group in a join of given groups, J. Austral. Math. Soc. 17 (1974), 434-495.

2. __ Nilpotent groups, Canad. Math. Congress Summer Seminar, Univ. of Alberta, 1957.

3. P. Neumann, Endomorphisms of infinite soluble groups, Bull. London Math. Soc. 12 (1980), 13-16.

4. J. M. Tyrer-Jones, Direct product and the Hopf property, J. Austral. Math. Soc. 17 (1974), 174-196.

5. J. S. Wilson, On characteristically simple groups, Math. Proc. Cambridge Philos. Soc. 80 (1976), 19-35.

Department of Mathematics, University of Alberta, Edmonton, Alberta, Canada 